\section{P2-208 EPIDEMIOLOGY OF MENTAL HANDICAP IN BAGHDAD}

doi:10.1136/jech.2011.142976j.41

T Muslat.* Health Authority, Abu-Dhabi, United Arab Emirates

Objectives To study epidemiology of mental handicap within 5 years in regard to sex, age of diagnosis, parents consanguinity, family history, degree of retardation, factors affecting patient's mother during pregnancy and delivery, affecting the patients during infancy and childhood, and the definite diagnosis.

Methods Retrospective study, data were drawn from records of 573 patients for 5 years, 261 from IRH and 312 from five institutions for handicapped.

Results Male/female: 2.1/1. 55.9\% from IRH were diagnosed at age $<6$ years. $36.5 \%$ mildly retarded, $49.4 \%$ moderate, and $14.1 \%$ severely retarded. $52.5 \%$ was the rate of consanguinity among the patient's parents. $33.3 \%$ had a positive family history. $15.7 \%$ of their mothers had disease or trauma during pregnancy, $10.7 \%$ had difficult, forceps or C.S delivery. $24.1 \%$ had infectious disease during infancy or early childhood, $18.2 \%$ had convulsions, $8.9 \%$ jaundice, and $7 \%$ trauma. Those with definite diagnosis (27.2\%), 8.6\% have mongolism, $7 \%$ post traumatic, $5.8 \%$ brain damage, and $2.8 \%$ had microcephaly, the remaining phenylketone urea, cretinism and cerebral palsy.

Conclusions There were predisposing factors promoting retardation in males, which suggested genetic influence. Only $55.9 \%$ were diagnosed in pre-school age. The high consanguinity of parents indicated the genetic factors operating on aetiology, still $47.5 \%$ had no family history which indicated the influence of specific environmental factors. Many of the diseases or complications during pregnancy, infancy and childhood were preventable or can be controlled. Mongolism had prevalence similar to other countries, so no racial difference. Most of patients was classified moderate retardation.

\section{P2-209 ASSOCIATION OF DIETARY FACTORS WITH BREAST DENSITY AMONG WOMEN IN SOUTH GERMANY}

doi:10.1136/jech.2011.142976j.42

${ }^{1} \mathrm{O}$ Voevodina, ${ }^{1} \mathrm{G}$ Nagel, ${ }^{*} \mathrm{C}$ Billich. ${ }^{1}$ Institute of Epidemiology and Medical Biometry, University of Ulm, Ulm, Germany; ${ }^{2}$ Departement of Diagnostic and Interventional Radiology, University of UIm, UIm, Germany

Introduction Effects of dietary factors on mammographic breast density are not sufficiently consistent to elaborate preventive recommendations. This study aims to investigate the association between current diet, alcohol consumption and mammographic density.

Methods We performed a cross-sectional study in 424 women aged 21-84 years. Current Mediterranean type dietary pattern, multivitamin-multimineral-supplementation, alcohol consumption and confounders were assessed with a self-administered questionnaire in the mammography unit of University Hospital Ulm (2008). Mammographic density was evaluated according to American College of Radiology classification and summarised in low (ACR1, 2) and high (ACR3, 4). Logistic regression models were calculated adjusted for age and BMI, menopausal status and other confounders. Results Adherence to Mediterranean type dietary pattern was inversely associated with high mammographic density, adjusted for age and BMI (per unit increase $\mathrm{OR}=0.95$; CI 0.90 to 0.997, $\mathrm{p}=0.41$ ). After stratification by menopausal status statistically significant association was present in post-menopausal women only. Current use of multivitamin-multimineral supplements was inversely associated with a high mammographic density in the adjusted model $(\mathrm{OR}=0.53$; CI 0.34 to $0.83, \mathrm{p}=0.01)$. Stratification by menopausal status shows similar associations, statistically significant only in post-menopausal women. Compared to non-drinkers excessive alcohol consumption (10-47 g/day) was in trend associated with higher mammographic density in the adjusted model (OR 1.47; CI 0.82 to $2.63, p=0.03$ )

Conclusion Adherence to Mediterranean type diet and current use of multivitamin-multimineral supplements may be associated with a lower mammographic density and may have a protective effect against breast cancer.

\section{P2-210 METABOLIC SYNDROME AND CERVICAL CANCER IN THE METABOLIC SYNDROME AND CANCER PROJECT (ME-CAN)}

doi:10.1136/jech.2011.142976j.43

${ }^{1} \mathrm{G}$ Nagel, ${ }^{* 2,3} \mathrm{~T}$ Bjorge, ${ }^{4} \mathrm{H}$ Concin, ${ }^{5} \mathrm{~A}$ Lukanova, ${ }^{6} \mathrm{~J}$ Manjer, ${ }^{7} \mathrm{G}$ Hallmans, ${ }^{7} \mathrm{H}$ Jonnson, ${ }^{7} \mathrm{P}$ Stattin, ${ }^{7,8} \mathrm{~T}$ Stocks, ${ }^{9} \mathrm{H}$ Ulmer. ${ }^{1}$ Ulm University, Ulm, Germany; ${ }^{2}$ University of Bergen, Bergen, Norway; ${ }^{3}$ Norwegian Institute of Public Health, Oslo, Bergen, Norway; ${ }^{4}$ Agency for Preventive and Social Medicine, Bregenz, Austria; ${ }^{5}$ German Cancer Research Center, Heidelberg, Germany; ${ }^{6}$ Skåne University Hospital Malmö, Malmö, Sweden; 7 Umeå University, Umeå, Sweden; ${ }^{8}$ Copenhagen University Hospital, Copenhagen, Denmark; ${ }^{9}$ Innsbruck Medical University, Innsbruck, Austria

Introduction Little is known about the association between the metabolic syndrome (MetS) and cervical cancer carcinogenesis.

Methods The Me-Can cohort includes 288834 women. During an average follow-up of 11 years 425 invasive cervical cancer cases were diagnosed. HRs were estimated by use of Cox proportional hazards regression models for quintiles and standardised $z$-scores (with a mean of 0 and a SD of 1) of body mass index, blood pressure, glucose, cholesterol, triglycerides and a MetS score. Risk estimates were corrected for random error in the measurements.

Results The MetS score was associated with increased risk of cervical cancer (per 1 SD increase, HR, 1.26; 95\% CI 1.08 to 1.47). Among individual metabolic factors, associations were observed for BMI (per 1 SD increase, 1.12; 1.01 to 1.25$)$, blood pressure $(1.25 ; 1.04$ to 1.49$)$, and triglycerides (1.39; 1.15 to 1.68$)$. In models including all metabolic factors simultaneously, the associations for blood pressure and triglycerides persisted. Stratification by morphology showed stronger association of triglycerides with squamous cell carcinoma (SCC) $(1.42 ; 95 \%$ CI1.09 to 1.84$)$ than with adenocarcinoma (ADC) $(0.97,0.53$ to 1.75$)$. Among older women cholesterol (50-70 years $\mathrm{HR}, 1.34 ; 95 \%$ CI 1.00 to 1.81$)$, triglycerides (50-70 years HR 1.49 , $95 \%$ CI 1.03 to 2.16 and $\geq 70$ years HR $1.53,95 \%$ CI 1.08 to 2.17 ) and glucose ( $\geq 70$ years HR $1.87,95 \%$ CI 1.12 to 3.12 ) concentrations were associated with cervical cancer.

Conclusions The results of this large prospective study provide evidence for an association between cervical cancer and the MetS as well as the individual MetS factors including BMI, blood glucose and triglyceride levels.

\section{P2-211 A TARGETED INTERVENTION TO PROMOTE BREAST CANCER SCREENING AMONG RURAL WOMEN IN COASTAL KARNATAKA, INDIA}

doi:10.1136/jech.2011.142976j.44

S Nair, ${ }^{*}$ M S Vidyasagar, S Kamath, S Nair. Manipal University, Manipal, Karnataka, India

Introduction Breast Cancer is the second common cancer among women in India and nearly $21 \%$ of the women, who develop the disease, die within the first year of diagnosis. Lack of awareness and facilities for early detection and treatment are some of the reasons for this. The rural field Practice area of a tertiary care teaching hospital is the setting for this study wherein a community based intervention was initiated to promote breast cancer screening 